\title{
A prospective study of lipoprotein(a) and risk of coronary heart disease among women with type 2 diabetes
}

Received: 11 October 2004 / Accepted: 9 March 2005 / Published online: 22 June 2005

(C) Springer-Verlag 2005

\begin{abstract}
Aims: We examined the association between lipoprotein (Lp)(a) and CHD among women with type 2 diabetes. Methods: Of 32,826 women from the Nurses' Health Study who provided blood at baseline, we followed 921 who had a confirmed diagnosis of type 2 diabetes. Results: During 10 years of follow-up (6,835 personyears), we documented 122 incident cases of CHD. After adjustment for age, smoking, BMI, glycosylated $\mathrm{HbA}_{1} \mathrm{c}$, triglycerides (TGs), high-density lipoprotein cholesterol, low-density lipoprotein cholesterol and other cardiovascular risk factors, the relative risk (RR) comparing extreme quintiles of $\mathrm{Lp}(\mathrm{a})$ was 1.95 (95\% CI 1.07-3.56). The association was not appreciably altered after further adjustment for apolipoprotein $\mathrm{B}_{100}$ or several inflammatory
\end{abstract}

I. Shai · M. B. Schulze · M. J. Stampfer · F. B. Hu

Department of Nutrition, Harvard School of Public Health, Boston, MA, USA

I. Shai $(\bowtie)$ - J. E. Manson · M. J. Stampfer · F. B. Hu Department of Epidemiology, Harvard School of Public Health, 677 Huntington Avenue,

Boston, MA, 02115, USA

e-mail: irish@bgu.ac.il; ishai@hsph.harvard.edu

Tel.: +11-972-86477447

Fax: +11-972-86477638

I. Shai

S. Daniel Abraham International Center for Health and Nutrition, Department of Epidemiology,

Ben-Gurion University,

Beer-Sheva, Israel

J. E. Manson · M. J. Stampfer · F. B. Hu

Division of Preventive Medicine, Department of Medicine, Brigham and Women's Hospital and Harvard Medical School, Boston, MA, USA

J. E. Manson · M. J. Stampfer · F. B. Hu Channing Laboratory, Department of Medicine, Brigham and Women's Hospital and Harvard Medical School, Boston, MA, USA

N. Rifai

Department of Laboratory Medicine, Children's Hospital and Harvard Medical School,

Boston, MA, USA biomarkers. Increasing levels of $\mathrm{Lp}$ (a) were associated with lower levels of TGs. The probability of developing CHD over 10 years was higher among diabetic women with substantially higher levels of both $\mathrm{Lp}(\mathrm{a})$ ( $>1.07$ $\mu \mathrm{mol} / \mathrm{l})$ and TGs $(>2.26 \mathrm{mmol} / \mathrm{l})$ than among diabetic women with lower levels (22 vs $10 \%, p$ log-rank test= 0.049). Diabetic women with a higher level of only $L p(a)$ or TGs had a similar (14\%) risk. In a multivariate model, diabetic women with higher levels of $L p(a)$ and TGs had an RR of 2.46 (95\% CI 1.21-5.01) for developing CHD, as compared with those with lower levels of both biomarkers ( $p$ for interaction $=0.413$ ). The RRs for women with a higher level of either $\mathrm{Lp}(\mathrm{a})(\mathrm{RR}=1.22,95 \% \mathrm{CI}$ $0.77-1.92)$ or $\mathrm{TGs}(\mathrm{RR}=1.39,95 \%$ CI $0.78-2.42)$ were comparable. Conclusions/interpretation: Increased levels of $L p(a)$ were independently associated with risk of CHD among diabetic women.

Keywords Coronary heart disease - Follow-up . Lipoprotein(a) - Type 2 diabetes

\begin{abstract}
Abbreviations apo: apolipoprotein $\cdot \operatorname{apoB}_{100}$ : apolipoprotein $\mathrm{B}_{100} \cdot \mathrm{CABG}$ : coronary artery bypass surgery - CRP: C-reactive protein - HDL-c: high-density lipoprotein cholesterol - LDL-c: low-density lipoprotein cholesterol - Lp: lipoprotein - MI: myocardial infarction . PTCA: coronary angioplasty $\cdot$ RR: relative risk $\cdot$ sICAM-1: soluble intercellular adhesion molecules - sTNF-RII: soluble TNF- $\alpha$ receptor $2 \cdot$ TGs: triglycerides
\end{abstract}

\section{Introduction}

Lipoprotein (Lp)(a) is a low-density lipoprotein (LDL)-like particle in which an apolipoprotein (apo)(a) moiety is linked via a disulphide bond to apolipoprotein $\mathrm{B}_{100}\left(\mathrm{apoB}_{100}\right)$ [1] A meta-analysis [2] identified Lp(a) as a modest risk factor for coronary events in the general population. The few small prospective studies conducted among diabetic patients have yielded various associations [3-5], whereas the prospective study that assessed the relationship between 
$\mathrm{Lp}(\mathrm{a})$ and cardiovascular disease did find a positive association [5].

The increased risk of CHD among diabetic women cannot be completely explained by the contribution of traditional risk factors. The most common pattern of dyslipidaemia in patients with type 2 diabetes is elevated triglycerides (TGs) and decreased high-density lipoprotein cholesterol (HDL-c) levels [6]. Cross-sectional studies among diabetic [7] and non-diabetic [8-10] populations have observed an inverse association between TGs and Lp(a) levels. It is not clear whether plasma TGs could play a role in $\mathrm{Lp}$ (a) metabolism and what is the joint effect of high TGs and Lp(a) levels on CHD.

Thus, we examined the association between Lp(a) and CHD and its relation with TGs among diabetic women in the Nurses' Health Study.

\section{Subjects, materials and methods}

Study population The Nurses' Health Study was initiated in 1976 with the enrolment of 121,700 US nurses aged $30-55$ years. This prospective cohort study is followed through biennially mailed questionnaires related to lifestyle factors and health outcomes. Of 32,826 study participants who provided blood samples in 1989-1990, 1194 had a confirmed diagnosis of type 2 diabetes at baseline or during follow-up until 1996. The present study included 921 women who did not report a diagnosis of myocardial infarction (MI), coronary bypass surgery (CABG), coronary angioplasty (PTCA) or stroke at the time blood was drawn and had complete biomarker data available. The study was approved by the institutional review boards at the Harvard School of Public Health and the Brigham and Women's Hospital; completion of the self-administered questionnaire was considered to imply informed consent.

Definition of diabetes Diagnosis of diabetes was reported by the participants on the biennial questionnaires. We mailed a supplementary questionnaire to all women reporting diabetes to obtain further information about the date of diagnosis, symptoms, diagnostic tests, and treatment for hyperglycaemia. In accordance with the criteria of the National Diabetes Data Group [11], confirmation of diabetes required at least one of the following self-reports on the supplementary questionnaire: (1) an elevated plasma glucose concentration (fasting plasma glucose $\geq 7.8 \mathrm{mmol} / \mathrm{l}$, random plasma glucose $\geq 11.1 \mathrm{mmol} / 1$, and/or plasma glucose $\geq 11.1 \mathrm{mmol} / 1$ after $\geq 2 \mathrm{~h}$ during an oral glucose tolerance test) plus at least one classic symptom (excessive thirst, polyuria, weight loss or hunger); (2) no symptoms but at least two elevated plasma glucose concentrations (by the above criteria) on different occasions; or (3) treatment with hypoglycaemic medication (insulin or oral hypoglycaemic agent). We used the National Diabetes Data Group criteria to define diabetes because our participants were diagnosed before the American Diabetes Association released their criteria in 1997 [12]. The validity of selfreported diagnosis of type 2 diabetes by the supplementary questionnaire has been established by a separate validation study through medical record reviews. Permission for medical record review was provided by 71 of 84 women, medical records were obtained for 62 , and the diagnosis of type 2 diabetes was confirmed in $98.4 \%$ [13].

CHD endpoints CHD endpoints consisted of fatal CHD, non-fatal MI and CABG/PTCA. Non-fatal MI was confirmed by reviewing medical records using the criteria of the World Health Organization of symptoms plus either typical electrocardiographic changes or elevated levels of cardiac enzymes [14]. Physicians who reviewed the records were blind to the self-reported risk-factor status. Deaths were reported by next of kin, the postal system, and through records of the National Death Index. With the use of all sources combined, the follow-up for deaths was more than $98 \%$ complete [15]. Fatal CHD was confirmed by review of medical records or autopsy reports with the permission of the next of kin. In no instance was the cause on the death certificate accepted without corroboration. Sudden deaths (i.e. death within $1 \mathrm{~h}$ of symptom onset in a woman without known disease that could explain death) were included in the fatal CHD category.

Blood collection and laboratory measurements Blood was collected during 1989 and 1990 from 32,826 participants, who were sent a blood-collection kit that included supplies (blood tubes, tourniquet, needles, bandage, coolant pack) and instructions. Participants arranged for the blood to be drawn and sent the samples back by prepaid overnight courier. Most samples arrived within $24 \mathrm{~h}$ of the blood being drawn. After arrival in the laboratory, samples were centrifuged and aliquoted into cryotubes as plasma, buffy coat and red blood cells. Cryotubes were stored in liquid nitrogen freezers at $-130^{\circ} \mathrm{C}$ or lower.

Measurements of lipid biomarkers were performed in the laboratory of Dr Nader Rifai (The Children's Hospital, Boston, MA, USA), which is certified by the NHLBI/CDC Lipid Standardization Program. All methods except the ELISA and RIA were done using a Hitachi 911 analyser (Roche Diagnostics, Indianapolis, IN, USA).

$\mathrm{Lp}$ (a) was measured by a latex-enhanced immunoturbidimetric method (Denka Sieken, Tokyo, Japan), with a CV of $2.6 \%$. This method is the only commercial assay that is not affected by the kringle IV type 2 repeats [16]. Total cholesterol was measured enzymatically with a $\mathrm{CV}$ of $<1.7 \%$. Concentrations of TGs and HDL-c were analysed simultaneously on the Hitachi 911, with CVs of 1.75 and $2.5 \%$, respectively. LDL cholesterol (LDL-c) was measured with a homogeneous direct method (Genzyme, Cambridge, MA, USA), with a CV of $<3.1 \%$. Total apoB ${ }_{100}$ was measured with an immunoturbidimetric technique on the Hitachi 911 analyser, with CVs of $<4.33 \%$. Fibrinogen was measured with an immunoturbidimetric assay on the Hitachi 911 analyser with reagents and calibrators from Kamiya Biomedical (Seattle, WA, USA) for testing an antigenantibody reaction and agglutination, with a CV of $1.16 \%$. Plasma was assayed for the presence of soluble TNFalpha receptor 2 (sTNF-RII) with the human sTNF-RII 
ELISA kit (R\&D Systems, Minneapolis, MN, USA), with a $\mathrm{CV}$ of $<5.1 \%$. Plasma C-reactive protein (CRP) was measured with the US CRP ELISA kit (DSL; Webster, TX, USA), with a CV of $<5.1 \%$. Since the CRP levels from the current assay consistently read higher than the CRP levels with an assay we used previously in our studies [17], we performed a cross-validation study using 204 samples obtained from the cohort of diabetic women whose CRP levels were measured by both methods. The correlation coefficient between the two methods was 0.97 , suggesting that the results reported for these assays should be comparable. Soluble intercellular adhesion molecules (sICAM1) were assayed in plasma with the human SICAM-1 ELISA kit (R\&D Systems), with a CV $<4.8 \%$. Plasma levels of sE-selectin were assayed with the human sEselectin ELISA kit (R\&D Systems), with a CV of $<8.8 \%$. Concentrations of glycosylated $\mathrm{HbA}_{1} \mathrm{c}$ [18] were based on turbidimetric immunoinhibition with haemolysed whole blood or packed red cells, with a $\mathrm{CV}$ of $<3.0 \%$.
Assessment of lifestyle exposures The women reported biennially on their lifestyle exposures. Alcohol intake was assessed with a validated dietary questionnaire in 1990, 1994 and 1998. A history of high blood pressure was determined from self-reports preceding blood collection. We calculated BMI as the ratio of weight $(\mathrm{kg})$ to the height squared $\left(\mathrm{m}^{2}\right)$, the latter being assessed in 1976.

Statistical analysis Accumulation of person-months of follow-up started in June 1990 and continued until June 2000. We used Cox proportional hazards analysis stratified on 5-year age categories and over each 2-year follow-up interval to estimate the relative risks (RR) for $L p(a)$ quintile compared with the lowest quintile. Participants who were diagnosed with CHD or stroke or died during follow-up were censored at the date of diagnosis or death. All other participants were followed through to June 2000. Tests for trend were calculated across the median values of increasing categories of biomarkers. We tested for effect modification by performing Cox proportional hazards an-
Table 1 Baseline age-adjusted characteristics of 921 women with type 2 diabetes across quintiles of $\mathrm{Lp}(\mathrm{a})$ : the Nurses' Health Study
Means or percentages, age-adjusted for the age groups $<49$, $50-54,55-59,60-64,65+$ years, except for age ${ }^{\top} n=622$; women who reported fasting more than $8 \mathrm{~h}$ before blood was drawn

${ }^{\ddagger}$ According to a regression between two CRP assays among subgroup of 204 diabetic women $(r=0.97, y=0.4+0.67 x)$ Mets, metabolic equivalents

\begin{tabular}{|c|c|c|c|c|c|c|}
\hline & \multicolumn{6}{|c|}{ Quintiles of $\mathrm{Lp}(\mathrm{a})$} \\
\hline & 1 & 2 & 3 & 4 & 5 & $p$ for trend \\
\hline Median $(\mu \mathrm{mol} / \mathrm{l})$ & 0.05 & 0.14 & 0.26 & 0.54 & 1.89 & \\
\hline Range & $0.0-0.09$ & $0.09-0.20$ & $0.20-0.36$ & $0.36-1.01$ & $1.01-6.30$ & \\
\hline Age (years) & 57.6 & 60.1 & 57.6 & 59.1 & 57.8 & 0.193 \\
\hline BMI $\left(\mathrm{kg} / \mathrm{m}^{2}\right)$ & 30.5 & 30.2 & 30.4 & 29.9 & 29.2 & 0.054 \\
\hline Current smokers (\%) & 16.9 & 14.5 & 10.5 & 12.9 & 13.8 & 0.869 \\
\hline Physical activity (Mets/week) & 27.9 & 27.6 & 48.2 & 33.5 & 18.9 & 0.245 \\
\hline Alcohol consumption (g/day) & 2.9 & 2.9 & 2.8 & 3.0 & 2.6 & 0.799 \\
\hline Daily aspirin use (\%) & 25.2 & 20.1 & 23.4 & 24.3 & 21.2 & 0.694 \\
\hline Postmenopausal hormone use (\%) & 31.5 & 26.9 & 24.8 & 25.1 & 26.8 & 0.640 \\
\hline Insulin use $(\%)$ & 17.4 & 15.9 & 19.9 & 20.7 & 22.4 & 0.188 \\
\hline Oral diabetic medication use (\%) & 21.2 & 22.8 & 14.0 & 21.8 & 19.7 & 0.955 \\
\hline $\begin{array}{l}\text { Cholesterol lowering medication } \\
\text { use }(\%)\end{array}$ & 4.2 & 3.7 & 5.4 & 4.8 & 1.6 & 0.109 \\
\hline Multivitamin supplement use (\%) & 39.0 & 38.5 & 35.4 & 38.6 & 36.2 & 0.716 \\
\hline Duration of type 2 diabetes (years) & 7.3 & 8.1 & 7.6 & 8.3 & 8.3 & 0.405 \\
\hline History of hypertension (\%) & 60.8 & 62.5 & 57.6 & 60.4 & 57.3 & 0.622 \\
\hline Parental history of MI (\%) & 23.6 & 19.5 & 19.9 & 24.0 & 26.4 & 0.144 \\
\hline Fasting status $(\%)$ & 66 & 62 & 65 & 72 & 69 & 0.324 \\
\hline \multicolumn{7}{|l|}{ Biomarkers } \\
\hline TGs (mmol/l) & 2.7 & 2.4 & 2.0 & 2.2 & 1.9 & $<0.0001$ \\
\hline Fasting $\mathrm{TGs}^{\dagger}(\mathrm{mol} / \mathrm{l})$ & 2.6 & 2.4 & 1.9 & 2.1 & 1.9 & $<0.0001$ \\
\hline HDL-c (mmol/l) & 1.3 & 1.3 & 1.4 & 1.3 & 1.4 & 0.040 \\
\hline LDL-c (mmol/l) & 3.4 & 3.5 & 3.6 & 3.8 & 3.7 & $<0.001$ \\
\hline $\mathrm{ApoB}_{100}(\mathrm{~g} / \mathrm{l})$ & 1.01 & 1.02 & 1.01 & 1.05 & 1.03 & 0.225 \\
\hline Total cholesterol/HDL-c ratio & 4.8 & 5.0 & 4.5 & 4.8 & 4.5 & 0.064 \\
\hline $\mathrm{HbA}_{1} \mathrm{c}(\%)$ & 6.8 & 6.8 & 6.8 & 6.8 & 7.0 & 0.185 \\
\hline Creatinine $(\mu \mathrm{mol} / \mathrm{l})$ & 68.1 & 68.9 & 68.9 & 68.9 & 68.9 & 0.652 \\
\hline sTNF-RII (pg/ml) & 2613.0 & 2488.0 & 2553.0 & 2636.0 & 2483.0 & 0.318 \\
\hline CRP (mg/l) & 8.7 & 6.9 & 8.0 & 8.1 & 9.0 & 0.172 \\
\hline Adjusted CRP ${ }^{\ddagger}$ (mg/l) & 5.4 & 4.3 & 5.0 & 5.0 & 5.6 & 0.172 \\
\hline sICAM-1 (ng/ml) & 327.3 & 316.4 & 306.2 & 320.4 & 313.0 & 0.647 \\
\hline sE-selectin (ng/ml) & 66.0 & 68.4 & 63.6 & 64.2 & 69.5 & 0.323 \\
\hline Fibrinogen $(\mu \mathrm{mol} / \mathrm{l})$ & 10.8 & 10.6 & 11.4 & 11.2 & 11.4 & 0.040 \\
\hline
\end{tabular}


alyses stratified by selected variables and by evaluating interaction terms that were computed by modelling the quintile medians as continuous variables. We performed Kaplan-Meier survival analysis to compare the CHD survival probabilities of women with combinations of $\mathrm{Lp}$ (a) levels $(\leq>>1.07 \mu \mathrm{mol} / 1$, suggested cutoff point [19] and near the top quintile among our study population) and TG levels $(\leq>>2.26 \mathrm{mmol} / 1$, cutoff point for pharmacological therapy among diabetic patients [6]). Finally, we evaluated the joint RR of $\mathrm{Lp}(\mathrm{a})(\leq>>1.07 \mu \mathrm{mol} / \mathrm{l})$ and $\mathrm{TG}(\leq />2.26$ $\mathrm{mmol} / \mathrm{l})$ levels in a multivariate model. All the variables that were included in the multivariate models were selected according to their established associations with $\mathrm{CHD}$ and their potential role as confounders. All statistical analyses were performed with SAS statistical software version 8.0 (SAS Institute, Cary, NC, USA).

\section{Results}

During up to 10 years of follow-up $(6,835$ person years; average follow-up 7.4 years), we documented 122 incident cases of CHD (46 non-fatal MI, 21 fatal CHD, 55 CABG/ PTCA) among 921 women with confirmed type 2 diabetes. Lp(a) levels were not materially associated with age, smoking, BMI or markers of inflammation or glycaemic control (Table 1). Levels of total or fasting TGs were similar in our study. Higher levels of Lp(a) were associated with lower levels of TGs and higher levels of LDL-c.

In a multivariate model (Table 2), adjusted for age, smoking, BMI, alcohol intake, physical activity, postmenopausal hormone use, aspirin use, parental history of MI, history of hypertension, and levels of $\mathrm{HbA}_{1} \mathrm{c}$, the RR of CHD for the extreme quintiles of $\mathrm{Lp}$ (a) was 1.76 (95\% CI $0.99-3.13 ; p$ for trend $=0.058$ ). The association was slightly stronger after further adjustment for TGs (extreme RR= $1.97,95 \%$ CI $1.10-3.55$; $p$ for trend $=0.030$ ) and remained similar after further adjustment in the final multivariable model for LDL-c and HDL-c (extreme RR $=1.95,95 \% \mathrm{CI}$ $1.07-3.56 ; p$ for trend $=0.035$ ). In a secondary analysis, when 245 participants who were diagnosed with diabetes after blood draw were excluded (106 remaining CHD cases), the final multivariate-adjusted RR comparing the extreme quintiles of $\mathrm{Lp}(\mathrm{a})$ remained similar $(\mathrm{RR}=2.00$, 95\% CI 1.03-3.89).

The association of $\mathrm{Lp}(\mathrm{a})$ with CHD was not appreciably changed after further adjustments in multivariate models for levels of $\operatorname{apoB}_{(100)}$, creatinine or fibrinogen, or for levels of CRP, sICAM-1, sE-selectin or sTNF-RII (data not shown).

Stronger associations between $\mathrm{Lp}(\mathrm{a})$ and CHD risk (Table 3) appeared to exist among non-aspirin users, nonusers of postmenopausal hormones, alcohol abstainers, diabetic women with longer duration of the disease, and diabetic women with higher levels of LDL-c or TGs. We observed no significant interactions in these multivariable models. However, the relatively small number of cases limited this stratified analysis.

The probability (Fig. 1) of developing CHD during the 10 years of follow-up was higher among diabetic women with higher levels of both $\mathrm{Lp}(\mathrm{a})(>1.07 \mu \mathrm{mol} / \mathrm{l})$ [19] and TGs $(>2.26 \mathrm{mmol} / \mathrm{l})[20]$ than among women with lower levels of these biomarkers (22\% vs $10 \%, p$ log-rank test=0.049). Diabetic women with a higher level of either $\mathrm{Lp}(\mathrm{a})$ or TGs had similar (14\%) risk. We examined the joint effect of $\mathrm{Lp}(\mathrm{a})$ and TGs in a multivariate model (Fig. 2), adjusted for several CHD risk factors and levels of $\mathrm{HbA}_{1} \mathrm{c}$, LDL-c and HDL-c. Diabetic women with higher levels of both Lp(a) and TGs had a RR of 2.46 (95\% CI 1.21-5.01) for developing CHD, compared with those with lower levels of both biomarkers. The RRs among women with a higher level of either Lp(a) or TGs were comparable ( $p$ for interaction $=0.413$ ).

\section{Discussion}

We found that, among women with type 2 diabetes, those with levels of $\mathrm{Lp}(\mathrm{a})$ in the highest quintile had twice the risk of CHD, independently of blood lipids, glycaemic control and markers of inflammation or other traditional CHD risk factors. $L p(a)$ levels were inversely associated with levels of TGs, but both biomarkers contributed independently to increased CHD risk.

Table 2 Relative risk (95\% CI) of CHD events across quintiles of Lp(a) among 921 women with type 2 diabetes: the Nurses' Health Study

\begin{tabular}{|c|c|c|c|c|c|c|}
\hline & \multicolumn{6}{|c|}{ Quintiles of Lp(a) } \\
\hline & 1 & 2 & 3 & 4 & 5 & $p$ for trend \\
\hline Median $(\mu \mathrm{mol} / \mathrm{l})$ & 0.05 & 0.14 & 0.26 & 0.54 & 1.89 & \\
\hline Cases $(n)$ & 22 & 19 & 27 & 24 & 30 & \\
\hline Age-adjusted & 1 & $0.03(0.01-0.05)$ & $0.04(0.02-0.08)$ & $0.04(0.02-0.07)$ & $0.05(0.03-0.08)$ & 0.145 \\
\hline $\mathrm{MV}^{*}$ & 1 & $0.04(0.02-0.07)$ & $0.06(0.03-0.10)$ & $0.04(0.02-0.07)$ & $0.06(0.03-0.11)$ & 0.058 \\
\hline MV+TGs & 1 & $0.04(0.02-0.07)$ & $0.06(0.03-0.12)$ & $0.04(0.02-0.08)$ & $0.07(0.04-0.13)$ & 0.030 \\
\hline MV+TGs+LDL-c+HDL-c & 1 & $0.04(0.02-0.07)$ & $0.06(0.03-0.12)$ & $0.04(0.02-0.08)$ & $0.07(0.04-0.13)$ & 0.035 \\
\hline
\end{tabular}

*Multivariate model, Cox regression, adjusted for age $(<49,50-54,55-59,60-64,65+$ years), smoking (current, past, never), BMI $(<23$, $\left.23-25,25-28,28-30,30-34,35+\mathrm{kg} / \mathrm{m}^{2}\right)$, alcohol intake $(0,0.1-4.9,5+\mathrm{g} /$ day $)$, physical activity $(0-0.9,1-1.9,2-3.9,4+\mathrm{h} / \mathrm{week})$, postmenopausal hormone use (premenopausal, current, past, never, missing), aspirin use (non-daily, daily), parental history of MI, history of hypertension and levels of $\mathrm{HbA}_{1} \mathrm{c}$ (quintiles)

MV, multivariate 


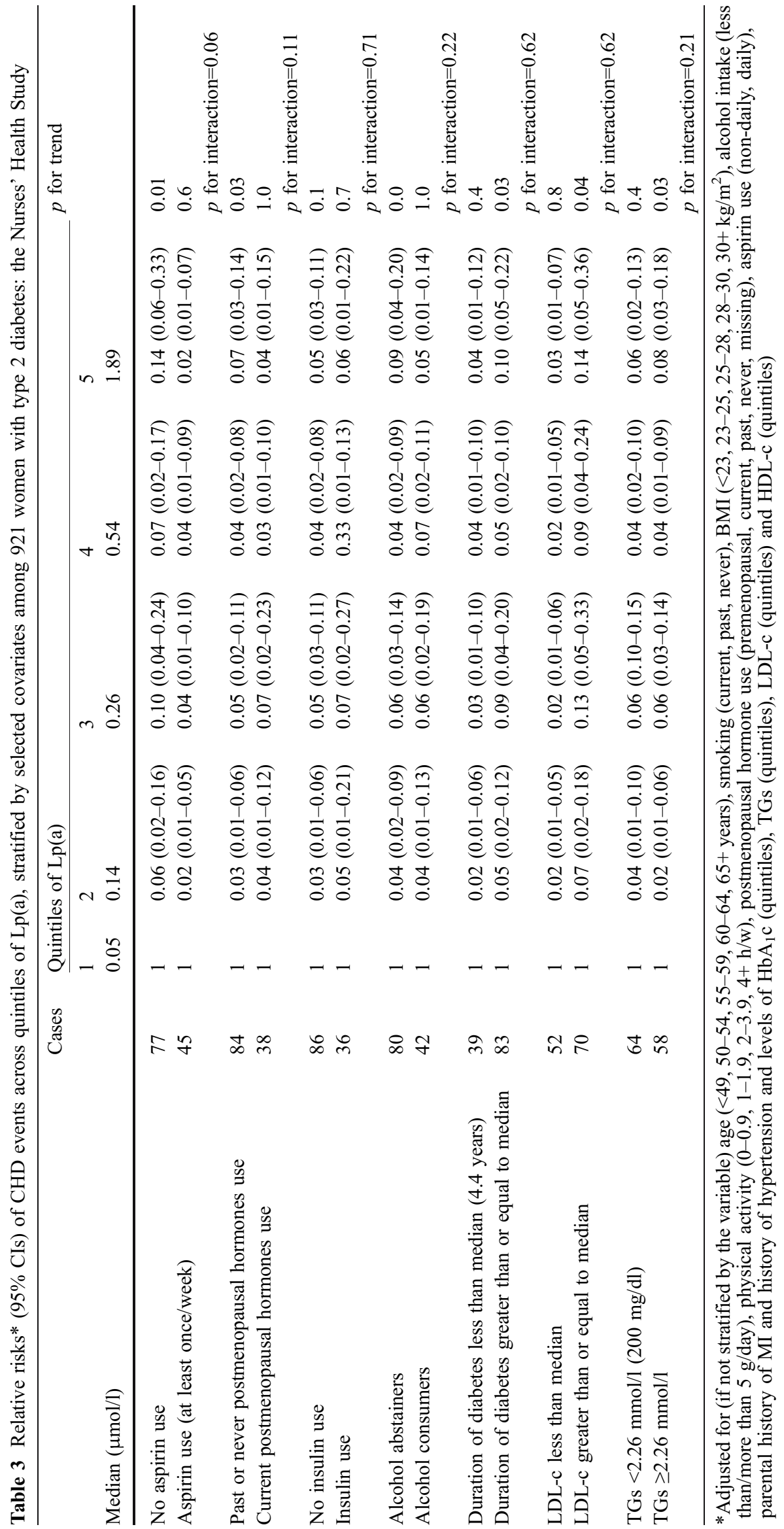


Fig. 1 CHD event-free survival across 10 years among 921 women with type 2 diabetes, stratified by risk combinations of Lp(a) and TGs: the Nurses' Health Study. Top line (shaded): $\mathrm{Lp}(\mathrm{a})<1.07 \mu \mathrm{mol} / 1, \mathrm{TGs}<2.26$ $\mathrm{mmol} / 1$ (46 cases among 444 women); second line from top (unbroken): $\mathrm{Lp}(\mathrm{a})<1.07 \mu \mathrm{mol} / 1$, TGs $>2.26 \mathrm{mmol} / 1$ (48 cases among 301 women); third line from top (broken): $\mathrm{Lp}(\mathrm{a})>1.07$ $\mu \mathrm{mol} / \mathrm{l}, \mathrm{TGs}<2.26 \mathrm{mmol} / \mathrm{l}(18$ cases among 130 women); bottom line (bold, unbroken): $\mathrm{Lp}(\mathrm{a})>1.07 \mu \mathrm{mol} / 1, \mathrm{TGs}>2.26$ $\mathrm{mmol} / \mathrm{l}$ (10 cases among 46 women)

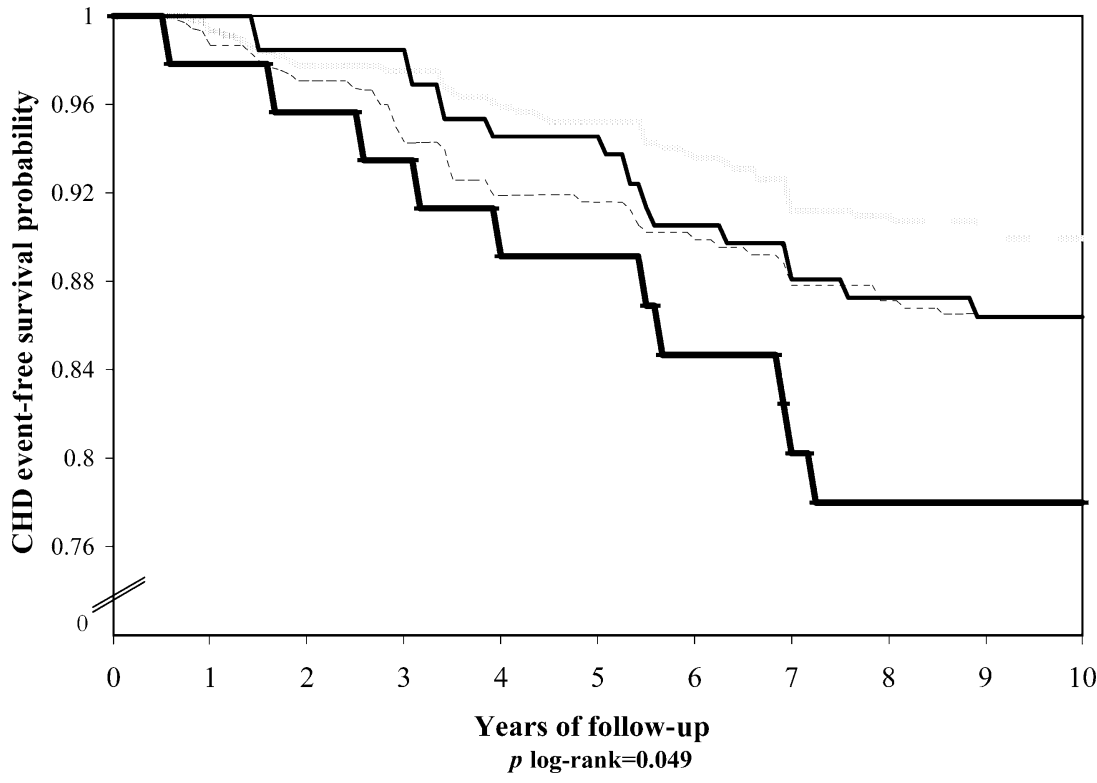

The strengths of the study include our well-established cohort with detailed and repeated measures of lifestyle exposures and the use of an advanced method of $L p(a)$ measurement; apo(a) varies in size from 300 to $800 \mathrm{kDa}$, depending on the number of plasminogen-like kringle IV type 2 repeats [21]. This size polymorphism is an important determinant of the density heterogeneity of $\operatorname{Lp}(\mathrm{a})$ [22], and is one of the significant sources of discrepancy among $\mathrm{Lp}(\mathrm{a})$ assays in the past. In this study we measured $\mathrm{Lp}(\mathrm{a})$ with a recently available assay that is not affected by plasminogen-like kringle IV type 2 repeats [16].

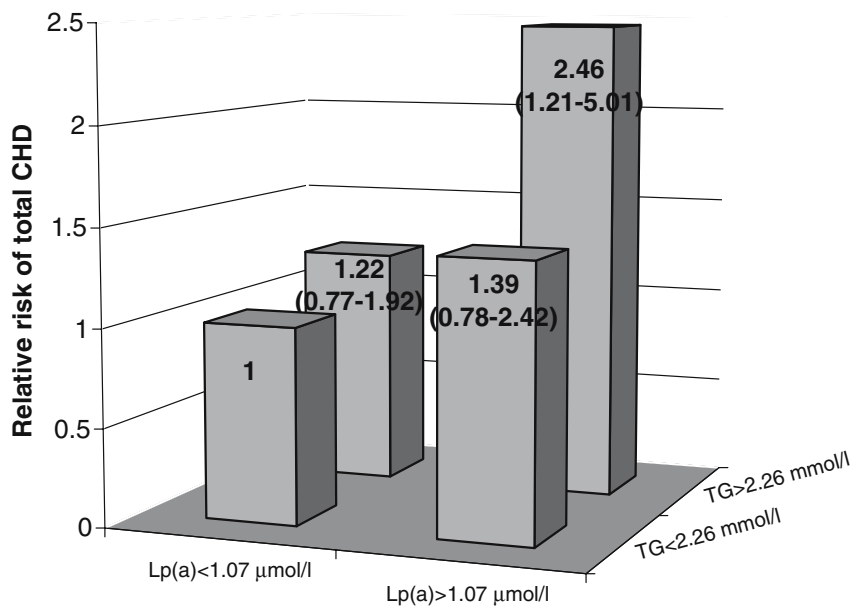

Fig. 2 Multivariate*-adjusted relative risk of CHD among women with type 2 diabetes according to baseline levels of $\mathrm{Lp}(\mathrm{a})$ and TGs: the Nurses' Health Study. $p$ for interaction $=0.413$. *Adjusted for age $(<49,50-54,55-59,60-64,65+$ years), smoking (current, past, never), BMI $\left(<23,23-25,25-28,28-30,30+\mathrm{kg} / \mathrm{m}^{2}\right)$, alcohol intake (less than or more than $5 \mathrm{~g}$ /day), physical activity $(0-0.9,1-1.9,2-$ $3.9,4+\mathrm{h} /$ week), postmenopausal hormone use (premenopausal, current, past, never, missing), aspirin use (non-daily, daily), parental history of MI, history of hypertension, and levels of $\mathrm{HbA}_{1} \mathrm{c}$ (quintiles), LDL-c (quintiles) and HDL-c (quintiles)
Our study has several limitations. First, single measurements of biomarkers may not reflect individual variation over time and the effects we have observed may be underestimates due to random measurement error. However, the apo(a) gene accounts for more than $90 \%$ of the variation in plasma $\mathrm{Lp}$ (a) concentrations [23]. Secondly, although we found most of our biomarkers to be stable if blood specimens were processed within $36 \mathrm{~h}$ of collection [24], Lp(a) appeared to be stable for up to only $24 \mathrm{~h}$. However, $97 \%$ of the samples were returned within $24 \mathrm{~h}$ of collection. Thirdly, measurement of $\mathrm{Lp}(\mathrm{a})$ in frozen samples is likely to result in falsely lower $\mathrm{Lp}$ (a) levels in samples with low molecular weight apo(a) isoforms [25]. This degradation, if present, may lead to underestimation of the associations we observed. Finally, results of analyses of $L p(a)$ lipoprotein vary according to race, ethnic origin and age. Our data are limited to women from the Nurses' Health Study, almost all of whom were white. Therefore, our data may not be applicable to other racial/ethnic groups.

Several small studies among patients with type 1 or 2 diabetes observed inconsistent results regarding $\mathrm{Lp}(\mathrm{a})$. In a 10 -year prospective study of 186 young patients with type 1 diabetes, higher levels of $\mathrm{Lp}(\mathrm{a})$ were not associated with macrovascular disease [4]. In a 6-month angiography follow-up of 102 patients with type 2 diabetes and after intracoronary stent placement, Lp(a) and apo(a) polymorphisms did not significantly predict restenosis [3]. In a prospective 2- to 3-year study of 221 patients with type 2 diabetes, the incidence of clinical events related to CHD was significantly higher in patients with high serum $L p(a)$ levels [5].

$\mathrm{Lp}$ (a) accumulates in the arterial wall at the sites of atherosclerotic lesions beyond its proportional levels in plasma [26]. In addition to its role as a cholesterol carrier, Lp(a) has a thrombogenic effect because of the structural homology of apo(a) and plasminogen [27]. Lp(a) enhances platelet aggregation [28], modulates vascular endothelial 
cell function [29] and is considered an acute-phase reactant [30]. It is not clear whether the mechanisms by which Lp(a) is involved in atherosclerosis progression in the diabetic population is identical to that in the non-diabetic population.

We found that $\mathrm{Lp}(\mathrm{a})$ and TGs were inversely associated and that the survival rate or the RR of CHD among women with TGs $>2.26 \mathrm{mmol} / 1$ was comparable to that among women with $\mathrm{Lp}(\mathrm{a})$ levels $>1.07 \mu \mathrm{mol} / \mathrm{l}$. This may suggest that, although elevated TGs are considered one of the most common patterns of dyslipidaemia in patients with type 2 diabetes [6], some patients with diabetes and lower TG levels may have elevated $\mathrm{Lp}(\mathrm{a})$ levels that could predict a similar risk of CHD. This hypothesis should be carefully explored further. A cross-sectional study among 69 patients with type 1 diabetes and 122 patients with type 2 diabetes [7] found an inverse and independent association between TGs and Lp(a), suggesting that plasma TGs could play a role in $\mathrm{Lp}$ (a) metabolism. As reviewed in that study [7], this inverse association could be due to accelerated clearance of apo(a) present in the TG-rich particles compared with the slower catabolism of apo(a) in the LDL density range.

Little is know about treatment affecting Lp(a). Optimisation of glycaemic control does not affect serum $\mathrm{Lp}(\mathrm{a})$ levels in patients with type 2 diabetes [31]; most of the cholesterol-lowering drugs are targeted to LDL-c and are less effective against $L p(a)$ [32]. Favourable effects on $\mathrm{Lp}$ (a) levels have been suggested for fish intake [33], the use of nicotinic acid [34] and the use of oestrogen or testosterone [35].

In conclusion, our results suggest that, among women with type 2 diabetes, increased levels of $\mathrm{Lp}(\mathrm{a})$ are associated with increased risk of CHD, independently of several CHD risk factors. The combination of high TGs and high $\mathrm{Lp}$ (a) may confer a particularly high risk of CHD among diabetic women.

Acknowledgements This study was supported by research grants CA87969, HL65582, HL34594 and DK58845 from the National Institutes of Health. We thank Dr Sue Hankinson for her invaluable contribution. Dr Hu is partially supported by an American Heart Association Established Investigator award. Dr Schulze is supported by a fellowship of the German Academic Exchange Service (DAAD). Dr Shai is supported by the Fulbright Foundation, USA, and by the S. Daniel Abraham International Center for Health and Nutrition, BenGurion University of the Negev, Israel.

\section{References}

1. Milionis HJ, Winder AF, Mikhailidis DP (2000) Lipoprotein (a) and stroke. J Clin Pathol 53:487-496

2. Danesh J, Collins R, Peto R (2000) Lipoprotein(a) and coronary heart disease. Meta-analysis of prospective studies. Circulation 102:1082-1085

3. Gazzaruso C, Garzaniti A, Falcone C et al (2003) Lipoprotein (a), apolipoprotein(a) polymorphism and restenosis after intracoronary stent placement in Type 2 diabetic patients. J Diabetes its Complicat 17:135-140
4. Maser RE, Usher D, Becker DJ et al (1993) Lipoprotein(a) concentration shows little relationship to IDDM complications in the Pittsburgh Epidemiology of Diabetes Complications Study cohort. Diabetes Care 16:755-758

5. Hiraga T, Kobayashi T, Okubo M et al (1995) Prospective study of lipoprotein(a) as a risk factor for atherosclerotic cardiovascular disease in patients with diabetes. Diabetes Care 18:241244

6. Haffner SM (2004) American Diabetes Association. Dyslipidemia management in adults with diabetes. Diabetes Care 27 [Suppl 1]:S68-S71

7. Hernandez C, Chacon P, Garcia-Pascual L, Simo R (2001) Differential influence of LDL cholesterol and triglycerides on lipoprotein(a) concentrations in diabetic patients. Diabetes Care 24:350-355

8. Bartens W, Rader DJ, Talley G, Brewer HB (1994) Decreased plasma levels of lipoprotein(a) in patients with hypertriglyceridemia. Atherosclerosis 108:149-157

9. Werba JP, Safa O, Gianfranceschi G, Michelagnoli S, Sirvatori CR, Franceschini G (1993) Plasma triglycerides and lipoprotein (a): inverse relationship in a hyperlipidemic Italian population. Atherosclerosis 101:203-207

10. Hiraga T, Okubo M, Koboyashi T, Nakanishi K, Sugimoto T, Murase T (1993) Serum lipoprotein(a) levels differ in different phenotypes of primary hyperlipoproteinemia. Metabolism 42:13271330

11. National Diabetes Data Group (1979) Classification and diagnosis of diabetes mellitus and other categories of glucose intolerance. Diabetes 28:1039-1057

12. Report of the Expert Committee on the Diagnosis and Classification of Diabetes Mellitus (1997) Diabetes Care 20:11831197

13. Manson JE, Rimm EB, Stampfer MJ et al (1991) Physical activity and incidence of non-insulin-dependent diabetes mellitus in women. Lancet 338:774-778

14. Rose GA, Blackburn H, Gillum RF, Prineas RJ (1982) Cardiovascular survey methods. World Health Organization, Geneva

15. Stampfer MJ, Willett WC, Speizer FE et al (1984) Test of the National Death Index. Am J Epidemiol 119:837-839

16. Marcovina SM, Albers JJ, Scanu AM et al (2000) Use of a reference material proposed by the International Federation of Clinical Chemistry and Laboratory Medicine to evaluate analytical methods for the determination of plasma lipoprotein(a). Clin Chem 46:1956-1967

17. Hu FB, Meigs JB, Li TY, Rifai N, Manson JE (2004) Inflammatory markers and risk of developing type 2 diabetes in women. Diabetes 53:693-700

18. Chang J, Hoke C, Ettinger B, Penerian G (1998) Evaluation and interference study of haemoglobin A1c measured by turbidimetric inhibition immunoassay. Am J Clin Pathol 109:274-278

19. Stein JH, Rosenson RS (1997) Lipoprotein Lp(a) excess and coronary heart disease. Arch Intern Med 157:1170-1176

20. Expert Panel on Detection Evaluation, and Treatment of High Blood Cholesterol in Adults (2001) Executive summary of the third report of the National Cholesterol Education Program (NCEP) expert panel on detection, evaluation, and treatment of high blood cholesterol in adults (Adult Treatment Panel III). JAMA 285:2486-2497

21. Scanu AM, Edelstein C (1965) Kringle-dependent structural and functional polymorphism of apolipoprotein(a). Biochim Biophys Acta 1256:1-12

22. Reblin T, Rader DJ, Beisiegel U, Greten H, Brewer HB Jr (1992) Correlation of apolipoprotein(a) isoproteins with $\mathrm{Lp}(\mathrm{a})$ density and distribution in fasting plasma. Atherosclerosis 94:223-232

23. Boerwinkle E, Leffert CC, Lin J, Lackner C, Chiesa G, Hobbs HH (1992) Apolipoprotein(a) gene accounts for greater than $90 \%$ of the variation in plasma lipoprotein (a) concentrations. J Clin Invest 90:52-60 
24. Pai JK, Curhan GC, Cannuscio CC, Rifai N, Ridker PM, Rimm EB (2002) Stability of novel plasma markers associated with cardiovascular disease: processing within 36 hours of specimen collection. Clin Chem 48:1781-1784

25. Kronenberg F, Trenkwalder E, Dieplinger H, Utermann G (1996) Lipoprotein(a) in stored plasma samples and the ravages of time. Why epidemiological studies might fail. Arterioscler Thromb Vasc Biol 16:1568-1572

26. Rath M, Niendorf A, Reblin T, Dietel M, Krebber HJ, Beisiegel U (1989) Detection and quantification of lipoprotein(a) in the arterial wall of 107 coronary bypass patients. Arteriosclerosis 9:579-592

27. Gaubatz JW, Heideman C, Gotto AM Jr, Morrisett JD, Dahlen GH (1983) Human plasma lipoprotein [a]: structural properties. J Biol Chem 258:4582-4589

28. Rand ML, Sangrar W, Hancock MA et al (1998) Apolipoprotein(a) enhances platelet responses to the thrombin receptoractivating peptide SFLLRN. Arterioscler Thromb Vasc Biol 18:1393-1399

29. Schachinger V, Halle M, Minners J, Berg A, Zeiher AM (1997) Lipoprotein(a) selectively impairs receptor-mediated endothelial vasodilator function of the human coronary circulation. J Am Coll Cardiol 30:927-934
30. Craig WY, Ledue TB (1992) Lipoprotein(a) and the acute phase response. Clin Chim Acta 210:231-232

31. Emanuele N, Azad N, Abraira C et al (1998) Effect of intensive glycemic control on fibrinogen, lipids, and lipoproteins: Veterans Affairs Cooperative Study in Type II Diabetes Mellitus. Arch Intern Med 158:2485-2490

32. Gonbert S, Malinsky S, Sposito AC et al (2002) Atorvastatin lowers lipoprotein(a) but not apolipoprotein(a) fragment levels in hypercholesterolemic subjects at high cardiovascular risk. Atherosclerosis 164:305-311

33. Marcovina SM, Kennedy H, Bittolo Bon G et al (1999) Fish intake, independent of apo(a) size, accounts for lower plasma lipoprotein(a) levels in Bantu fishermen of Tanzania: the Lugalawa Study. Arterioscler Thromb Vasc Biol 19:1250-1256

34. Carlson LA, Hamsten A, Asplund A (1989) Pronounced lowering of serum lipid levels of lipoprotein(a) in hyperlipidemic subjects treated with nicotinic acid. J Intern Med 226:271-276

35. Berglund L, Carlström K, Stege R et al (1996) Hormonal regulation of serum lipoprotein(a) levels: effects of parenteral administration of estrogen or testosterone in males. J Clin Endocrinol Metab 81:2633-2637 